\title{
Chlamydia pneumoniae in coronary and iliac arteries of Japanese patients with atherosclerotic cardiovascular diseases
}

\author{
KAZUNOBU OUCHI, BANYO FUJII*, YASUO KANAMOTO†, MIKIO KARITA $\$$, MUTSUNORI \\ SHIRAI $\ddagger$ and TERUKO NAKAZAWA $\ddagger$
}

Departments of Paediatrics and *Internal Medicine, Saiseikai Shimonoseki General Hospital, 3-4-1 Kifune-cho, Shimonoseki, Yamaguchi-751, †Department of Nutrition. Hiroshima Chuo Women's Junior College, Hiroshima and + Department of Microbiology, Yamaguchi University School of Medicine, Ube, Japan

\begin{abstract}
Recent studies suggest the association of atherosclerotic cardiovascular diseases with Chlamydia pneumoniae infection in western populations. It is of great interest whether such an association exists in Asians with their distinct genetic background. Symptomatic patients with coronary heart disease (29) or arteriosclerosis obliterans (10) who underwent directional endo-atherectomy were studied. Atherectomy specimens of coronary and iliac arteries were examined for $C$. pneumoniae by culture, nested PCR and immunohistochemical stain (IHC) with one Chlamydia genus-specific, two $C$. pneumoniae species-specific, and two $C$. trachomatis species-specific monoclonal antibodies. Among the 29 patients with coronary artery disease, $C$. pneumoniae was detected in the coronary arteries of 13 by IHC, 16 by PCR and 20 by IHC or PCR, or both. $C$. pneumoniae was also found in the iliac arteries of four patients by IHC, three by PCR and five by IHC or PCR, or both, of the 10 patients with arteriosclerosis obliterans. Attempts to isolate $C$. pneumoniae by culture were unsuccessful. The re-stenotic rate after atherectomy was higher in the $C$. pneumoniae-positive group than in the negative group, but not significantly so. These findings support the high incidence of $C$. pneumoniae in atherosclerotic lesions of symptomatic patients with coronary heart disease and arteriosclerosis obliterans in Asians.
\end{abstract}

\section{Introduction}

Chlamydia pneumoniae is the third species of the genus Chlamydia and is known as a leading cause of human acute respiratory tract infections worldwide [1]. C. pneumoniae frequently causes sporadic or epidemic community-acquired respiratory infections, such as pharyngitis, sinusitis, bronchitis and pneumonia. Recent sero-epidemiological evidence suggests an association between chronic $C$. pneumoniae infection and several diseases, such as asthma [2,3], sarcoidosis [4] and atherosclerosis [5-12].

The association of $C$. pneumoniae with atherosclerosis in sero-epidemiological studies is based on the demonstration of elevated IgG or IgA antibody titres to $C$. pneumoniae, or both, in patients with coronary

Received 18 Nov. 1997; revised version accepted 21 Jan. 1998.

Corresponding author: K. Ouchi. heart disease, asymptomatic carotid atherosclerosis and ischaemic cerebrovascular disease. All of these studies have shown the adjusted Odds ratio for the association of $C$. pneumoniae with such atherosclerotic categories to be c. 2.0 [5-12]. Furthermore, several laboratories have succeeded in detecting $C$. pneumoniae in coronary and aortic atheroma samples, but not in normal arteries, by immunohistochemical staining (IHC), polymerase chain reaction (PCR) and electron microscopy [13-18]. However, there is controversy about the presence of $C$. pneumoniae in atherosclerotic lesions [19]. Whether $C$. pneumoniae has a direct causative role in the development of atherosclerosis also remains to be elucidated. Hitherto, all seroepidemiological studies as well as studies demonstrating $C$. pneumoniae in atheroma have been made in western countries. Further data are required from other populations, such as from Asians, to consolidate this observation. The aim of the present study was to detect $C$. pneumoniae in atherectomy specimens from symptomatic patients with coronary artery disease and arteriosclerosis obliterans in a Japanese population. 


\section{Materials and methods}

\section{Subjects and tissues examined}

Thirty-nine Japanese patients (aged 45-78 years; 27 males and 12 females) with ischaemic heart disease or arteriosclerosis obliterans treated consecutively by a first atherectomy from September 1993 to March 1994 at Saiseikai Shimonoseki General Hospital, Shimonoseki, Japan were enrolled in this study. The characteristics of these patients are shown in Table 1. All patients had stenotic lesions of the coronary or iliac arteries of $>90 \%$. Atherosclerotic plaques resected by directional endo-atherectomy were tested for the presence of $C$. pneumoniae by IHC with monoclonal antibody, PCR and culture. Serum samples were obtained from 25 of the 39 patients for chlamydial serology at the time of atherectomy and stored at $-80^{\circ} \mathrm{C}$ until tested. Patients were monitored for the occurrence of re-stenosis until August 1997.

Additionally, when the proximal end of the venous graft was anastomosed to the ascending aorta, small pieces of the aortic lesions were removed by punch biopsy from 12 patients (aged 57-65 years) who were undergoing coronary artery by-pass surgery (CABS) from January 1994 to September 1994 and were examined by PCR and IHC. Although macroscopically less advanced atherosclerotic lesions were chosen for anastomoses, four of the aortic punched-out samples were classified as having intimal thickening and eight as having mild atheromatous plaques. Coronary artery samples of 10 paediatric patients who died of asphyxia, drowning or bacterial meningitis (age range 1 day -2 years) and two biopsy samples of the aorta from patients with aortic regurgitation (aged 19 and 22 years) were used as non-atherosclerotic controls.

Atherectomy specimens were immediately placed into Chlamydia transport medium SPG [20] and frozen at $-80^{\circ} \mathrm{C}$ for isolation and PCR or into buffered formalin $10 \%$ for IHC.

\section{$I H C$}

After paraffin-embedding, all samples were sliced into $5-\mu \mathrm{m}$ sections and stained by immuno-peroxidase by the avidin-biotin-peroxidase method with the DACO LSAB kit (DACO, Glostrup, Denmark). Slides of each specimen were stained with the following five monoclonal antibodies (MAbs): CF-2, a genus-specific MAb to chlamydial lipopolysaccharide (Washington Research Foundation, Seattle, USA) at 1 in 1000 dilution; two C. pneumoniae-specific MAbs, RR-402 (Washington Research Foundation) and AY-6 (53 kD outermembrane protein-specific, Hitachi Chemical, Tokyo, Japan), both at a 1 in 1000 dilution; and two $C$. trachomatis-specific MAbs, KK-12 (Washington Research Foundation) and 1651 (MOMP specific, Virostat, ME, USA), both at a 1 in 100 dilution. Control slides with HEp-2 cell monolayers infected with $C$. pneumoniae or C. trachomatis were run. in parallel with each staining batch.

\section{PCR}

Two resected lesions were homogenised with a new tissue homogeniser for each patient and centrifuged at $15000 \mathrm{rpm}$ for $30 \mathrm{~min}$. Pellets were resuspended in $100 \mu \mathrm{l}$ of PCR buffer containing $50 \mathrm{mM} \mathrm{KCl}, 10 \mathrm{mM}$ Tris-Cl, pH 8.3, NP40 $0.5 \%$, Tween $200.5 \%$ with proteinase $\mathrm{K} 1 \mathrm{mg} / \mathrm{ml}$ and incubated at $55^{\circ} \mathrm{C}$ for $1 \mathrm{~h}$. After the reaction mixture was heated at $95^{\circ} \mathrm{C}$ for $10 \mathrm{~min}$, DNA was extracted twice with equal volumes of phenol-chloroform-isoamyl alcohol and precipitated with isopropanol by standard methods and dissolved in $100 \mu \mathrm{l}$ of $\mathrm{H}_{2} \mathrm{O}$ [21]. The nested PCR test to detect the C. pneumoniae-specific 474-bp PstI fragment reported by Campbell et al. [22] was used in this study. The first PCR was done with the HL-1 and HR-1 primer set and the second PCR by the newly designed primer set, ON1, 5'-TTGAGCATATTCGTGAGG-3'; ON-2, 5'-GTACAGTTTCTCCGTTAG- $3^{\prime}$. The sizes of the products of each PCR were $437 \mathrm{bp}$ and $190 \mathrm{bp}$. The PCR amplification mixture contained $0.5 \mu \mathrm{M}$ primers, $100 \mu \mathrm{M}$ dNTPs, $5 \mathrm{mM} \mathrm{MgCl}_{2}$ and $1 \mathrm{U}$ Taq DNA polymerase (Takara, Kyoto, Japan) for the first and the second PCR. Twenty $\mu$ l of sample were used for the first PCR and $2 \mu \mathrm{l}$ of the first PCR product for the second PCR. The samples were denatured initially at $94^{\circ} \mathrm{C}$ for $5 \mathrm{~min}$ and the mixtures were subjected to 30 thermal cycles of denaturation at $94^{\circ} \mathrm{C}$ for $1 \mathrm{~min}$, annealing at $55^{\circ} \mathrm{C}$ for $1 \mathrm{~min}$ and extension at $72^{\circ} \mathrm{C}$ for $1 \mathrm{~min}$ with a 5-min additional extension for the last cycle. PCR products were evaluated by DNA size and restriction pattern with $A c c \mathrm{I}$ digestion. This PCR technique allows the detection of $5.0 \times 10^{-3}$ ifu for C. pneumoniae strain TW-183.

\section{Isolation by culture}

The resected atherosclerotic plaques were homogenised in SPG with a tissue homogeniser and inoculated on to a HEp-2 cell monolayer in 96-well microtitration plates by centrifugation at $1700 \mathrm{~g}$ for $1 \mathrm{~h}$ followed by cycloheximide $(1 \mu \mathrm{g} / \mathrm{ml})$ treatment as described previously [23]. After incubation for $72 \mathrm{~h}$ at $35^{\circ} \mathrm{C}$, plates were fixed and stained by the indirect fluorescent antibody technique with $C$. pneumoniae-specific $\mathrm{MAb}$ RR-402. At least three cell passages were done for each specimen.

\section{Serology}

Titres of IgG, IgA and IgM antibody to C. pneumoniae were measured in serum by a micro-immunofluorescence test [24]. Formalinised whole elementary bodies of $C$. pneumoniae strain TW-183 were used as an antigen. 


\section{Data collection and analysis}

All data were verified by a retrospective review of the patient records. Statistical comparisons were performed by the $\chi^{2}$ test with Yates' correction for categorical variables. Fisher's exact test was used when the minimum estimated value was $<5$. Continuous variables were analysed with the Mann-Whitney test. A p value $<0.05$ was considered significant.

\section{Results}

Demographic data for the atherosclerotic patients undergoing a first atherectomy are listed in Table 1. The only apparent difference in clinical characteristics between patients with coronary artery disease and those with arteriosclerosis obliterans was the greater rate of habitual smoking in the latter, although this and other differences were not statistically significant. C. pneumoniae was detected in $55 \%$ (16 of 29) by PCR and $46 \%$ ( 13 of 28 ) by IHC of atherectomy specimens from coronary arteries of patients with ischaemic heart disease. Of the samples from patients with atherosclerosis obliterans of iliac arteries, C. pneumoniae was demonstrated in $30 \%$ ( 3 of 10 ) by PCR and $40 \%$ ( 4 of 10 ) by IHC. Overall, C. pneumoniae was detected in $69 \%$ (20 of 29) of atherosclerotic coronary arteries and in $50 \%$ ( 5 of 10) of atherosclerotic iliac arteries by either PCR or IHC, or both (Table 2). All attempts to isolate $C$. pneumoniae by culture were unsuccessful. Of the 12 punch samples of aortic lesions obtained during
CABS, one $(8 \%)$ was positive for C. pneumoniae by PCR and IHC. This C. pneumoniae-positive sample was classified as a mild atherosclerotic plaque. None of the 10 control samples from paediatric patients or of the two aortic biopsy samples was positive by IHC or PCR or both. Representative results of IHC are shown in Fig. 1.

In IHC no discrepant results among the three MAbs (CF-2, RR-402, and AY-6) were observed. All samples were negative in IHC with two C. trachomatis speciesspecific MAbs.

Serum IgG, IgA and IgM antibodies against $C$. pneumoniae were positive in $25(100 \%), 7(28 \%)$ and none $(0 \%)$, respectively, of the 25 patients who were studied serologically. The mean and range of $\operatorname{IgG}$ serum titres of $C$. pneumoniae were 280 and 32-4096, respectively, and 10 patients $(40 \%)$ had $\operatorname{IgG}$ titres $\geqslant 512$. The range of IgA serum titres in seven positive patients was 8-64. The rate of positivity for antibodies to $C$. pneumoniae did not differ significantly between patients positive or negative for $C$. pneumoniae in their coronary or iliac arteries.

The clinical characteristics and distribution of risk factors for atherosclerosis according to positivity for C. pneumoniae in atherectomy specimens are listed in Table 3. The rate of re-stenosis after atherectomy was higher in the C. pneumoniae-positive group (13 of 25 , $52 \%$ ) than in the negative group (4 of $14,29 \%)$, although this difference was not statistically significant

Table 1. Characteristics of 39 patients undergoing atherectomy in the present study

\begin{tabular}{lccc}
\hline Characteristic & Coronary heart disease & Arteriosclerosis obliterans & $\mathrm{p}$ \\
\hline Number of patients & 29 & 10 \\
Gender (male: female) & $19: 10$ & $8: 2$ \\
Age: mean (range) & $65(47-78)$ & $64(45-73)$ \\
Indication for atherectomy (n) & Unstable angina pectoris (14) & Arteriosclerosis obliterans (10) \\
Stenotic lesion (n) & Myocardial infarction (15) & Iliac artery (10) \\
Cardiovascular risk factor (percent of & Right coronary artery (7) & & \\
patients) & & & NS \\
Hypertension & 62 & 20 & NS \\
Hyperlipidaemia & 38 & 20 & NS \\
Diabetes mellitus & 31 & 80 & NS \\
Habitual smoking & 55 & 60 & NS \\
Family history of CAD & 68 & & \\
\hline
\end{tabular}

CAD, coronary artery disease; NS, not significant.

Table 2. Results of detection of C. pneumoniae from atherectomy specimens of atherosclerotic lesions

\begin{tabular}{lcccc}
\hline & \multicolumn{4}{c}{ Number of positive results/number tested (\%) } \\
\cline { 2 - 6 } Sample tested & PCR & IHC & PCR or IHC or both & Culture \\
\hline Atherectomy specimens of & $16 / 29(55)$ & $13 / 28(46)$ & $20 / 29(69)$ & $0 / 18(0)$ \\
$\quad$ coronary heart disease $(\mathrm{n}=29)$ & $3 / 10(30)$ & $4 / 10(40)$ & $5 / 10(50)$ & $0 / 6$ \\
$\quad$ arteriosclerosis obliterans $(\mathrm{n}=10)$ & $1 / 12(8)$ & $1 / 12(8)$ & $1 / 12(8)$ & NT \\
Punch biopsies of aortic lesions in patients for CABS & $0 / 2(0)$ & $0 / 12(0)$ & $0 / 12(0)$ & NT \\
Control $^{*}(\mathrm{n}=12)$ & &
\end{tabular}

IHC, immunohistochemistry; CABS, coronary artery bypass surgery; NT, not tested.

${ }^{*}$ Control, autopsy samples of coronary arteries from 10 paediatric patients and two biopsy specimens of aorta from patients aged 19 and 22 years with aortic regurgitation as control without atherosclerosis 

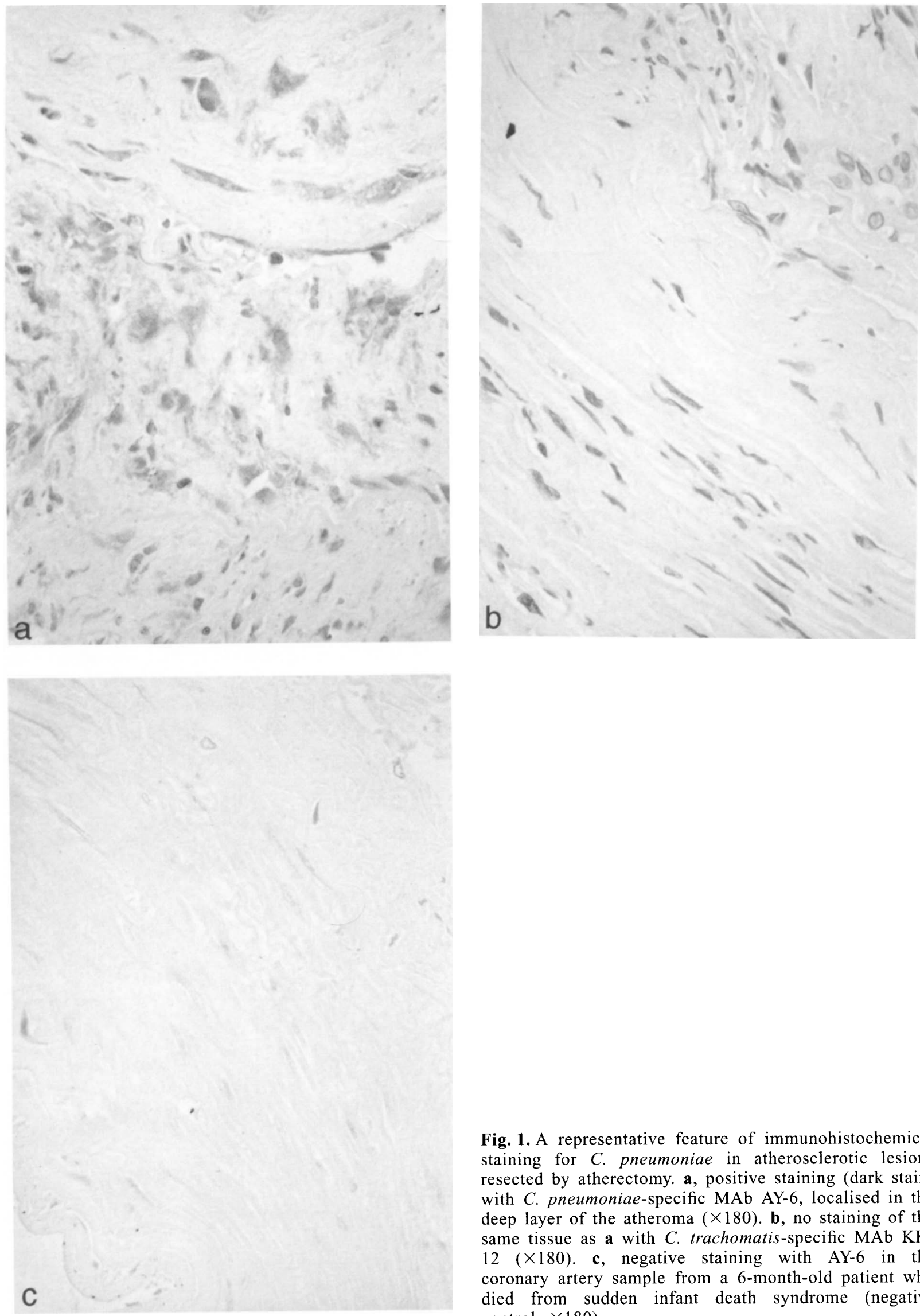

Fig. 1. A representative feature of immunohistochemical staining for $C$. pneumoniae in atherosclerotic lesions resected by atherectomy. a, positive staining (dark stain) with $C$. pneumoniae-specific MAb AY-6, localised in the deep layer of the atheroma $(\times 180)$. b, no staining of the same tissue as a with $C$. trachomatis-specific MAb KK$12(\times 180)$. c, negative staining with AY-6 in the coronary artery sample from a 6-month-old patient who died from sudden infant death syndrome (negative control, $\times 180$ ).

$(\mathrm{p}=0.10)$. Clinical factors such as age, sex, habitual smoking, hyperlipidaemia, hypertension, a family history of coronary artery disease and serum IgG or

IgA antibody titres against $C$. pneumoniae did not affect significantly the incidence of tissue positivity for C. pneumoniae in atherectomy specimens. A 
Table 3. Demographic data and distribution of risk factors for atherosclerosis of patients with or without $C$. pneumoniae in atherectomy specimens

\begin{tabular}{|c|c|c|c|}
\hline Parameter & $\begin{array}{l}\text { C. pneumoniae positive group }(\mathrm{n}=25) \text { : } \\
\text { number }(\%)\end{array}$ & $\begin{array}{c}\text { C. pneumoniae negative group }(\mathrm{n}=14) \text { : } \\
\text { number }(\%)\end{array}$ & $\begin{array}{c}p \\
\text { value }\end{array}$ \\
\hline Mean age (SD) & $63.9(6.6)$ & $65.4(10.9)$ & NS \\
\hline Male/female & $20 / 5$ & $7 / 7$ & NS \\
\hline $\begin{array}{l}\text { IgG antibody to } C \text {. pneumoniae } \\
\text { geometric mean titre (SD) }\end{array}$ & $1: 294(1: 184)$ & $1: 256(1: 128)$ & NS \\
\hline \multicolumn{4}{|l|}{ Diagnosis } \\
\hline Unstable angina & $12(48)$ & $7(50)$ & NS \\
\hline Post-myocardial infarction & $8(32)$ & $2(14)$ & NS \\
\hline Arteriosclerosis obliterans & $5(20)$ & $5(36)$ & NS \\
\hline Re-stenotic lesion & $13(52)$ & $4(29)$ & NS \\
\hline Habitual smoking & $17(68)$ & $7(50)$ & NS \\
\hline Hypertension & $17(68)$ & $8(57)$ & NS \\
\hline Hyperlipidaemia & $10(40)$ & $3(21)$ & NS \\
\hline Diabetes mellitus & $10(40)$ & $1(7)$ & $<0.05$ \\
\hline Family history of CAD & $17(68)$ & $8(57)$ & NS \\
\hline
\end{tabular}

NS, not significant; CAD, coronary artery disease.

statistically significant positive association was noted only between the rate of diabetes mellitus and a positive tissue test for $C$. pneumoniae.

\section{Discussion}

There is controversy about the presence of $C$. pneumoniae in atheroma of coronary arteries [19]. The results of this study support its presence in atherosclerotic lesions of coronary and iliac arteries in symptomatic patients. The rate of tissue positivity for C. pneumoniae in this study, i.e., $69 \%$ in atherosclerotic coronary arteries and $50 \%$ in atherosclerotic large arteries in Japanese patients, is comparable to the $51-79 \%$ found in previous studies of western populations [13-18]. This is the first report of positive results of atherosclerotic tissue tests for $C$. pneumoniae in Asians, whose seropositive rate by age is similar to that in western populations [25]. Furthermore, the positivity rate by PCR and IHC for C. pneumoniae of aortic atherosclerotic lesions from patients undergoing CABS was lower $(8 \%)$ than that of atherectomy specimens of symptomatic patients in the present study $(p=0.01)$. In addition, the degree of atherosclerosis in the lesions was less.

The control tissue specimens examined were not agematched to the case specimens in this study, although none was $C$. pneumoniae-positive. In a previous study with age- and sex-matched coronary artery segments from normal young adults, most of the atheromatous plaques were positive for $C$. pneumoniae, but no normal control coronary artery was positive [26]. These findings provide evidence for a possible involvement of $C$. pneumoniae in the pathogenesis of atherosclerosis in the coronary and iliac arteries.

Several discrepant results of PCR and IHC were observed in the present study. Of 25 C. pneumoniaepositive samples, 11 were positive by both PCR and
IHC, eight by PCR only and six by IHC only. The same discrepancy was shown in previous studies $[14,26]$. This discrepancy may be caused by the use of different pieces of atherectomy samples for each test, because it has been reported that C. pneumoniae is present in localised areas of a lesion [26]. Another possibility is that several specimens may contain PCR inhibitors, despite attempts to remove them by phenolchloroform DNA extraction.

C. pneumoniae could not be isolated by culture from atherectomy samples, even though fresh atherectomy specimens were frozen and handled by the same methods that have been used successfully to isolate the organism from nasopharyngeal specimens. So far, failure to isolate $C$. pneumoniae from coronary arteries has been reported in two studies. Autopsy samples were used in one [15] and atherectomy samples in the other [19]. All studies, including the present study, failed to isolate a living organism. However, Ramirez et al. recently reported the isolation of a strain of $C$. pneumoniae from an atherosclerotic coronary artery of a fresh explanted heart of a patient undergoing heart transplant [27]. $\gamma$-Interferon, tumour necrosis factor and nutrition deprivation induce persistent, non-productive chlamydial infection [28]. Furthermore, even though it has been established that in $C$. trachomatis infections, chlamydial antigen or DNA is sometimes detected by non-cultural diagnostic tests that do not depend on chlamydial viability, viable C. trachomatis organisms cannot be isolated from children with moderate to severe active trachoma [29]. The present study used one genus-specific and two species-specific MAbs for IHC and obtained exactly the same results. As the three MAbs are directed against different outer-membrane epitopes, the outer membrane of $C$. pneumoniae would seem to be intact. It is possible that most of the $C$. pneumoniae in atherosclerotic lesions may be present in a persistent, non-productive form and only a small portion in a productive, culturable form. 
In a South African study, Puolakkainen et al. [30] reported that $C$. pneumoniae antigen and DNA were detectable in coronary arterial atherosclerotic lesions more frequently in patients with low IgG titres against C. pneumoniae than in those with higher IgG titres. In both the Seattle study [14] and the present Japanese study, $C$. pneumoniae IgG antibody titres did not significantly affect the incidence of a positive tissue result for $C$. pneumoniae. Although the reason for this discrepancy is unknown, further studies are needed to elucidate whether $C$. pneumoniae serology may provide guidelines for the presence of $C$. pneumoniae in atherosclerotic lesions.

Campbell et al. [14] suggested a higher $C$. pneumoniae-positive rate in re-stenotic tissues, but Muhlestein et al. [17] found a lower re-stenosis rate in $C$. pneumoniae-positive tissues, although the differences were not statistically significant in these studies. These lesions had not been examined for evidence of $C$. pneumoniae before the development of re-stenosis. In contrast, the present study examined native lesions and then followed the patients for subsequent re-stenosis for 3-4 years. A higher, but not significantly higher, re-stenosis rate was found in patients with a $C$. pneumoniae-positive test $(\mathrm{p}=0.10)$. Although atherectomy has been replaced by percutaneous transluminal coronary angioplasty with stent implantation, further prospective studies of large numbers of samples will be required to clarify this finding.

An earlier sero-epidemiological study did not find any association between $C$. pneumoniae seropositivity and coronary heart disease in diabetic patients [31]. However, a statistically significant positive association was noted in the present study between the rate of diabetes mellitus and a positive tissue test for $C$. pneumoniae. It is well known that host defences are impaired in patients with diabetes mellitus. Abnormalities in adherence [32], chemotaxis [33], phagocytosis [32] and intracellular oxidative killing [34] contribute to the impaired function. Therefore, host defences against $C$. pneumoniae, a gram-negative intracellular bacterium, might be weak in diabetic patients and frequent or persistent $C$. pneumoniae infections may occur.

In conclusion, these results give further support to the presence of $C$. pneumoniae in atherosclerotic lesions of coronary and large arteries in symptomatic patients with atherosclerotic cardiovascular diseases. Further studies are required to clarify the direct causative role of C. pneumoniae in the pathogenesis of atherosclerosis.

\section{References}

1. Kuo CC, Jackson LA, Campbell LA, Grayston JT. Chlamydia pneumoniae (TWAR). Clin Microbiol Rev 1995; 8: 451-461.
2. Hahn DL, Dodge RW, Golubjatnikov R. Association of Chlamydia pneumoniae (strain TWAR) infection with wheezing, asthmatic bronchitis, and adult-onset asthma. JAMA 1991; 266: 225-230.

3. Emre U, Roblin PM, Gelling $\mathrm{M}$ et al. The association of Chlamydia pneumoniae infection and reactive airway disease in children. Arch Pediatr Adolesc Med 1994; 148: 727-732.

4. Black CM, Bullard JC, Staton GW, Hutwagner LC, Perez RL. Seroprevalence of Chlamydia pneumoniae antibodies in patients with pulmonary sarcoidosis in north central Georgia. Proc Eur Soc Chlamydia Res 1992; 2: 175 A.

5. Linnanmäki E, Leinonen $M$, Mattila K, Nieminen MS, Valtonen V, Saikku P. Chlamydia pneumoniae-specific circulating immune complexes in patients with chronic coronary heart disease. Circulation 1993; 87: 1130-1134.

6. Melnick SL, Shahar E, Folsom AR et al. Past infection by Chlamydia pneumoniae strain TWAR and asymptomatic carotid atherosclerosis. Atherosclerosis Risk in Communities (ARIC) Study Investigators. Am J Med 1993; 95: 499-504.

7. Patel P, Mendall MA, Carrington D et al. Association of Helicobacter pylori and Chlamydia pneumoniae infections with coronary heart disease and cardiovascular risk factors. $B M J$ 1995; 311: $711-714$.

8. Saikku P, Leiononen M, Mattila K et al. Serological evidence of an association of a novel Chlamydia, TWAR, with chronic coronary heart disease and acute myocardial infarction. Lancet 1988; 2: 983-986.

9. Saikku P, Leinonen M, Tenkanen L et al. Chronic Chlamydia pneumoniae infection as a risk factor for coronary heart disease in the Helsinki Heart Study. Ann Intern Med 1992; 116: $273-278$.

10. Thom DH, Wang SP, Grayston JT et al. Chlamydia pneumoniae strain TWAR antibody and angiographically demonstrated coronary artery disease. Arterioscler Thromb 1991; 11: $547-551$.

11. Thom DH, Grayston JT, Siscovick DS, Wang S-P, Weiss NS, Daling JR. Association of prior infection with Chlamydia pneumoniae and angiographically demonstrated coronary artery disease. JAMA 1992; 268: 68-72.

12. Wimmer MLJ, Sandmann-Strupp R, Saikku P, Haberl RL. Association of Chlamydial infection with cerebrovascular disease. Stroke 1996; 27: 2207-2210.

13. Blasi F, Denti F, Erba $M$ et al. Detection of Chlamydia pneumoniae but not Helicobacter pylori in atherosclerotic plaques of aortic aneurysms. $J$ Clin Microbiol 1996; 34: 2766-2769.

14. Campbell LA, O'Brien ER, Cappuccio AL et al. Detection of Chlamydia pneumoniae TWAR in human coronary atherectomy tissues. J Infect Dis 1995; 172; 585-588.

15. Kuo C-C, Shor A, Campbell LA, Fukushi H, Patton DL, Grayston JT. Demonstration of Chlamydia pneumoniae in atherosclerotic lesions of coronary arteries. J Infect Dis 1993; 167: $841-849$.

16. Kuo CC, Gown AM, Benditt EP, Grayston JT. Detection of Chlamydia pneumoniae in aortic lesions of atherosclerosis by immunocytochemical stain. Arterioscler Thromb 1993; 13: 1501-1504.

17. Muhlestein JB, Hammond EH, Carlquist JF et al. Increased incidence of Chlamydia species within the coronary arteries of patients with symptomatic atherosclerotic versus other forms of cardiovascular disease. J Am Coll Cardiol 1996; 27: 15551561 .

18. Shor A, Kuo CC, Patton DL. Detection of Chlamydia pneumoniae in coronary arterial fatty streaks and atheromatous plaques. S Afr Med J 1992; 82: 158-161.

19. Weiss SM, Roblin PM, Gaydos CA et al. Failure to detect Chlamydia pneumoniae in coronary atheromas of patients undergoing atherectomy. J Infect Dis 1996; 173: 957-962.

20. Nash P, Krenz MM. Culture media. In: Balows A, Hausler WJ, Herrmann KL, Isenberg HD, Shadomy HJ (eds) Manual of clinical microbiology, 5th edn. Washington, DC, American Society for Microbiology. 1991: 1226-1288.

21. Wilson K. Preparation of genomic DNA from bacteria. In: Ausubel FM, Brent R, Kringston RE et al. (eds) Current protocols in molecular biology. New York, Greene Publishing and Wiley-Interscience. 1991: 2.4.1-2.4.2

22. Campbell LA, Melgosa MP, Hamilton DJ, Kuo C-C, Grayston JT. Detection of Chlamydia pneumoniae by polymerase chain reaction. J Clin Microbiol 1992; 30: 434-439.

23. Roblin PM, Dumornay W, Hammerschlag MR. Use of HEp-2 
cells for improved isolation and passage of Chlamydia pneumoniae. J Clin Microbiol 1992; 30: 1968-1971.

24. Wang SP, Kuo CC, Grayston IT. Formalinized Chlamydia trachomatis organisms as antigen in the micro-immunofluorescence test. J Clin Microbiol 1979; 10: 259-261.

25. Kanamoto Y, Ouchi K, Mizui M, Ushio M, Usui T. Prevalence of antibody to Chlamydia pneumoniae TWAR in Japan. J Clin Microbiol 1991; 29: 816-818.

26. Kuo CC, Grayston JT, Campbell LA, Goo YA, Wissler RW, Benditt EP. Chlamydia pneumoniae (TWAR) in coronary arteries of young adults (15-34 years old). Proc Natl Acad Sci USA 1995; 92: 6911-6914.

27. Ramirez JA and the Chlamvdia pneumoniae/Atherosclerosis Study Group. Isolation of Chlamydia pneumoniae from coronary artery of a patient with coronary atherosclerosis. Ann Intern Med 1996; 125: 979-982.

28. Beatty WL, Byrne GI, Morrison RP. Repeated and persisten infection with Chlamydia and the development of chronic inflammation and disease. Trends Microbiol 1994; 2: 94-98.

29. Schachter J, Moncada J, Dawson CR et al. Nonculture methods for diagnosing chlamydial infection in patients with trachoma: a clue to the pathogenesis of the disease? J Infect Dis 1988; 158: $1347-1352$

30. Puolakkainen M, Kuo C-C, Shor A, Wang S-P, Grayston JT, Campbell LA. Serological response to Chlamydia pneumoniae in adults with coronary arterial fatty streaks and fibrolipid plaques. J Clin Microbiol 1993; 31: 2212-2214.

31. Miettinen $\mathrm{H}$, Lehto $\mathrm{S}$, Saikku $\mathrm{P}$ et al. Association of Chlamydia pneumoniae and acute coronary heart disease events in non-insulin dependent diabetic and non-diabetic subjects in Finland. Eur Heart $J$ 1996; 17: 682-688.

32. Bagdade JD, Root RK, Bulger RJ. Impaired leukocyte function in patients with poorly controlled diabetes. Diabetes $1974 ; 23$ : $9-15$.

33. Mowat A, Baum J. Chemotaxis of polymorphonuclear leukocytes from patients with diabetes mellitus. $N$ Engl J Med 1971; 284: $621-627$.

34. Wilson RM, Tomlinson DR, Reeves WG. Neutrophil sorbitol production impairs oxidative killing in diabetes. Diabetic Med $1987 ; 4: 37-40$. 